\title{
Long-Lasting Enhancement of Glutamatergic Synaptic Transmission by Acetylcholine Contrasts with Response Adaptation after Exposure to Low-Level Nicotine
}

\author{
Romain Girod and Lorna W. Role \\ Department of Anatomy and Cell Biology, Center for Neurobiology and Behavior, Columbia University, College of \\ Physicians and Surgeons, New York, New York 10032
}

\begin{abstract}
Attempts to mimic synaptic delivery of acetylcholine (ACh) with brief, repetitive pulses of high concentration $\mathrm{ACh}$ at synapses of medial habenula (MHN) and interpeduncular nucleus (IPN) neurons in vitro elicited temporally distinct facilitation and inhibition of glutamate secretion via nicotinic and muscarinic ACh receptor-mediated pathways, respectively. ACh-induced nicotinic facilitation was sustained for up to $2 \mathrm{hr}$, whereas muscarinic inhibition was transient. Prolonged exposure to nicotine inactivated nicotinic receptors selectively, thus decreasing the
\end{abstract}

The cholinergic system in the brain has been implicated in a variety of behavioral and cognitive functions, such as working memory, aspects of learning, attention, and arousal. These actions underlie the interaction of the endogenous neurotransmitter ACh with a variety of receptors that modulate neuronal excitability in networks that receive cholinergic afferents (Wainer et al., 1993; Sarter and Bruno, 1997). However, the cellular mechanisms underlying this neuromodulation are poorly understood. Although muscarinic acetylcholine receptors (mAChRs) are renowned for their effects in cortical regions affected in Alzheimer's disease, nicotinic acetylcholine receptors (nAChRs) may also contribute to cholinergic signaling in the normal and aging brain. Nicotine alters a variety of cognitive and behavioral functions through its specific interaction with $\mathrm{nAChRs}$ found within the diffuse terminal fields of central cholinergic projections (Woolf, 1991; Levin, 1992). Activation of $\mathrm{nAChRs}$ in circuits related to behavioral reinforcement may underlie the renowned effects of nicotine as an addictive drug (Stolerman and Shoaib, 1991; Schelling, 1992; Stolerman and Jarvis, 1995; Rose and Corrigall, 1997; Mansvelder and McGehee, 2000).

Nicotinic receptors are found in the cell bodies, dendrites, and within the presynaptic domains of neurons. Recent electrophysiological studies have provided direct evidence that nAChRs mediate synaptic transmission at central synapses (for review, see Jones et al., 1999). In addition, nAChRs are targeted to synaptic terminal and preterminal domains, consistent with demonstrated

Received Dec. 15, 2000; revised April 24, 2001; accepted May 1, 2001.

This work was supported by National Institute on Drug Abuse Awards DA09366 (to L.W.R.) and DA05840 (to R.G.). We thank A. B. MacDermott, E. K. Kandel, J. Koester, M. Jareb, Y. Jo, C. Du, P. Devay, and T. Davis for helpful suggestions for this manuscript. We also thank J. Goldberg for assistance in performing some of the initial experiments.

Correspondence should be addressed to L. W. Role, Department of Anatomy and Cell Biology, Center for Neurobiology and Behavior, Columbia University, College of Physicians and Surgeons, 1051 Riverside Drive, Psychiatric Institute Annex 807, New York, NY 10032. E-mail: lwr1@columbia.edu.

Copyright (C) 2001 Society for Neuroscience 0270-6474/01/215182-09\$15.00/0 relative contribution of the facilitatory versus inhibitory influences of ACh. The net effect of ACh in modulating glutamatergic transmission at MHN-IPN synapses may be determined by pre-exposure to nicotine, because the drug appears to switch the balance between the facilitatory and inhibitory actions of ACh.

Key words: neuromodulation; glutamate; acetylcholine; nicotine; presynaptic; nicotinic acetylcholine receptors; medial habenula; interpeduncular nucleus

effects of $\mathrm{ACh}$ and nicotine on the release of a wide variety of neurotransmitters (Rapier et al., 1990; Grady et al., 1992; McGehee et al., 1995; Dani and Heinemann, 1996; Gray et al., 1996; McGehee and Role, 1996; Role and Berg, 1996; Wonnacott, 1997; MacDermott et al., 1999).

Nicotine interaction with nAChRs facilitates the induction of long-term potentiation of glutamatergic neurotransmission in the hippocampus (Fujii et al., 1999), as well as in the ventral tegmental area (Mansvelder and McGehee, 2000). Whether similar mechanisms involving the activation of nicotinic receptors underlie the CNS effects of the endogenous ligand $\mathrm{ACh}$ remains to be determined. In addition, little is known of how prolonged exposure to nicotine might alter the net effect of $\mathrm{ACh}$ at central synapses in which transmission normally involves activation of both nicotinic- and muscarinic receptor-mediated pathways.

In this study we investigated the modulation of glutamate secretion by $\mathrm{ACh}$ and nicotine at synapses between medial habenula (MHN) and interpeduncular nucleus (IPN) neurons in vitro. The MHN-IPN pathway is part of the limbic system thought to be involved in arousal and reinforcement (Wilson et al., 1972; Wirtshafter, 1981). Glutamate serves as the excitatory transmitter at MHN-IPN synapses (Brown et al., 1983; McGehee et al., 1995). We used different patterns of nicotinic receptor agonist delivery in an attempt to mimic the modulation of cholinoceptive MHN-IPN synapses in the brain. Our results suggest that nicotine obtained from tobacco and ACh released at synaptic sites evoke distinct forms of neuromodulation of glutamate release that differ in both duration and magnitude. Furthermore, prolonged exposure to nicotine that mimicked habitual smoking inactivated nAChRs, thus profoundly altering the neuromodulatory effects of the endogenous transmitter.

\section{MATERIALS AND METHODS}

Cocultures. MHN and IPN were dissected from embryonic day 11 (E11) to E13 chicken embryos. Dispersed IPN neurons were cocultured with same-age MHN microexplants. Methods were as described previously 
(Role, 1988; Gardette et al., 1991; Brussaard et al., 1994), except that the MHN microexplants were plated $24 \mathrm{hr}$ before the IPN neurons.

Electrophysiology. Cells were visualized on a phase-contrast inverted microscope (Zeiss IM 135 TV; Zeiss, Oberkochen, Germany). The cultures were continuously suprafused with recording medium with a four-port perfusion nozzle. The external solution included (in mM): 145 $\mathrm{NaCl}, 2.5 \mathrm{KCl}, 0-1 \mathrm{MgCl}_{2}, 2.5 \mathrm{CaCl}_{2}$, and 10 HEPES, pH 7.4. An IPN neuron, visually confirmed to be contacted by processes emerging from the MHN microexplants, was selected and membrane currents were measured using the whole-cell perforated-patch configuration (Hamill et al., 1981) with amphotericin-permeabilized patches as described previously (Horn and Marty, 1988; Rae et al., 1991). The intracellular solution included: $53 \mathrm{~mm} \mathrm{KCl}, 75 \mathrm{~mm} \mathrm{~K}_{2} \mathrm{SO}_{4}, 5 \mathrm{mM} \mathrm{MgCl}_{2}, 10 \mathrm{~mm}$ HEPES, and $200 \mu \mathrm{M}$ amphotericin, $\mathrm{pH}$ 7.2. Voltage-clamp recordings were performed using a List EPC-7 patch-clamp amplifier (Medical Systems Corp., Greenvale, NY). The spontaneous activity was recorded in extracellular solution supplemented with tetrodotoxin (TTX) $(1 \mu \mathrm{M}$; Calbiochem, La Jolla, CA) to block $\mathrm{Na}^{+}$action potentials. Membrane currents were recorded continuously with a videocassette recorder (Sony, Tokyo, Japan) through a PCM interface (Digidata 1200; Axon Instruments, Foster City, CA). The currents were digitized with the pClamp 7.0 software (Axon Instruments). Spontaneous synaptic currents (minis) were detected from the digitized traces, and their amplitude, rise time, decay time, and frequency were computed with the Mini Analysis Program (Synaptosoft Inc., Leonia, NJ).

Drug application. The agonists and antagonists were applied by switching the perfusion medium with a solenoid valve-activated delivery system. Control experiments were performed by switching the regular medium to a medium diluted 10 -fold with water and by measuring the resulting change of junction potential at the tip of a patch electrode immersed in the recording bath. It was found that complete exchange of medium at the site of recording occurred within $<0.5 \mathrm{sec}$. Nicotine and acetylcholine were obtained from Sigma (St. Louis, MO).

Effects of prolonged exposure to nicotine were studied by adding the drug to established synaptic cocultures of MHN-IPN (1 $\mu \mathrm{M}$ nicotine, 1-3 d after coculture). After $24-72 \mathrm{hr}$, the treatment media was removed, and electrophysiological recordings were performed in control solution. Results obtained with 24 and $72 \mathrm{hr}$ nicotine treatment did not differ significantly and, as such, these data were pooled. Recovery from prolonged exposure to nicotine examined synaptic cocultures exposed previously to $1 \mu \mathrm{M}$ nicotine for $48 \mathrm{hr}$, after repeated exchange with 10 times the incubation volume by nicotine-free culture medium. Cultures were subsequently maintained for 3-24 hr in control, MHN-IPN conditioned media (Gardette et al., 1991).

Statistical assay of synaptic facilitation. Statistical methods were used to identify synapses that manifested significant changes in transmission after application of either ACh or nicotine and to compute the time course and magnitude of synaptic facilitation. The mini frequency was sampled for each innervated neuron as the number of minis occurring during sequential periods of $10 \mathrm{sec}$. The frequency bins were normalized to the average frequency calculated from at least $5 \mathrm{~min}$ of control recording time before agonist application. The average \pm SEM of three or six of these frequency bins was calculated to measure the average frequency during the initial, 1 min or longer intervals after experimental treatment. Significance of changes in mini frequency were compared for equal sample size and were evaluated by Student's $t$ test. Such analysis provided an unbiased assay of whether a synapse was cholinoceptive, and, if so, of the time at which mini frequency was significantly changed from baseline (delay for onset of facilitation), as well as of the time at which the frequency returned to the basal level (duration of facilitation).

\section{RESULTS}

\section{Synaptic facilitation by nicotinic AChRs-mediated pathways}

Patch-clamp recordings of IPN neurons were used to monitor synaptic activity at MHN-IPN synapses in vitro. The recordings were performed in the presence of TTX $(1 \mu \mathrm{M})$, sufficient to block the occurrence of superthreshold activity. The remaining TTXresistant synaptic activity was abolished by concurrent treatment with the glutamate receptor antagonists CNQX and APV (McGehee et al., 1995). Thus, the synaptic currents recorded in TTX-treated MHN-IPN preparations reflected the spontaneous secretion of glutamate (i.e., minis). Before application of ACh or nicotine, the basal mini frequency was approximately one to two events per second. The average amplitude of miniature synaptic currents was $10-20 \mathrm{pA}$ (holding potential of $-70 \mathrm{mV}$ ), with rising phases $<3.0 \mathrm{msec}$ and decay rates fit by single exponentials with $\tau$ values of $\sim 3-6 \mathrm{msec}$. Consistent with some well established features of spontaneous release, increasing extracellular tonicity or adjusting extracellular $\mathrm{K}^{+}$to $40 \mathrm{~mm}$ enhanced the frequency but not the amplitude of minis recorded (data not shown).

To investigate the neuromodulatory effects of ACh and nicotine on glutamate release, we monitored agonist-induced changes in the frequency of minis at MHN-IPN synapses. After a period of control recording of $>5-15 \mathrm{~min}$, the natural delivery of ACh as it likely occurs in vivo was approximated by applying shortduration, repetitive pulses of high concentrations of $\mathrm{ACh}$ to sites of neuron-neuron contact (100 msec, $50-200 \mu \mathrm{M} \mathrm{ACh,} 7$ pulses at $0.2 \mathrm{~Hz}$ ). ACh was removed between applications by local microperfusion in $<0.5 \mathrm{sec}$. In contrast, the more prolonged and low levels of nicotine, such as those achieved in arterial blood during smoking, were modeled by applying low concentrations of nicotine $(0.5-1 \mu \mathrm{M})$ continuously for $1 \mathrm{~min}$ (Benowitz et al., 1989). Both application paradigms elicited statistically significant modulation of synaptic transmission at a subset of MHN-IPN synapses (cholinoceptive synapses). Phasic application of ACh elicited a significant facilitation of TTX-resistant activity at 58\% of MHN-IPN synapses (total number of responsive synapses divided by the total number of connections recorded), whereas significant inhibition of transmission was recorded at $\sim 20 \%$ of the synapses $(n=24)$. In contrast, the effects of nicotine were strictly facilitatory ( $\sim 40 \%$ of tested synapses; $n=44)$ (Fig. 1$)$. The observation that $\mathrm{ACh}$ and nicotine did not elicit significant changes in mini frequency at all of the MHN-IPN synapses tested, as well as the range of effects elicited by cholinergic agonists at responsive synapses (see below), suggest that the population of synapses assayed is not homogeneous (McGehee et al., 1995). As such, our criteria for significance in cholinoceptive responses may result in an underestimate in the actual number of responsive synapses.

Synaptic current analysis before and after either nicotine or ACh treatment revealed that they were no significant changes in mini amplitude at the majority of synapses with statistically significant changes in mini frequency (data not shown). This finding is consistent with previous reports of nAChR-mediated presynaptic facilitation rather than a change in postsynaptic responses to glutamate (McGehee et al., 1995; Alkondon et al., 1996; Gray et al., 1996; Guo et al., 1998). Facilitation produced by the phasic application of ACh was typically larger in magnitude and more persistent than the synaptic facilitation elicited by continuous nicotine (Fig. 1, compare $A, B$ ).

Temporal differences between the responses to ACh and nicotine application were the most obvious (Fig. 2). The delay for onset of facilitation (i.e., the time elapsed between the beginning of agonist application and the time at which a statistically significant increase in the mini frequency was detected) was typically longer for ACh than for nicotine. Thus, with nicotine, $83 \%$ of facilitated synapses responded during application of the drug. In contrast, with $\mathrm{ACh}$, less than half of the cholinoceptive synapses exhibited significant changes in mini frequency within $1 \mathrm{~min}$ of agonist application (Fig. 2Ai). The average delay in onset of facilitation was $0.7 \pm 0.2 \mathrm{~min}$ with nicotine versus an average delay of $1.5 \pm 0.5 \mathrm{~min}$ with ACh (Fig. $2 \mathrm{Bi}$ ). The difference in average delay observed with the two agonists was statistically significant. 


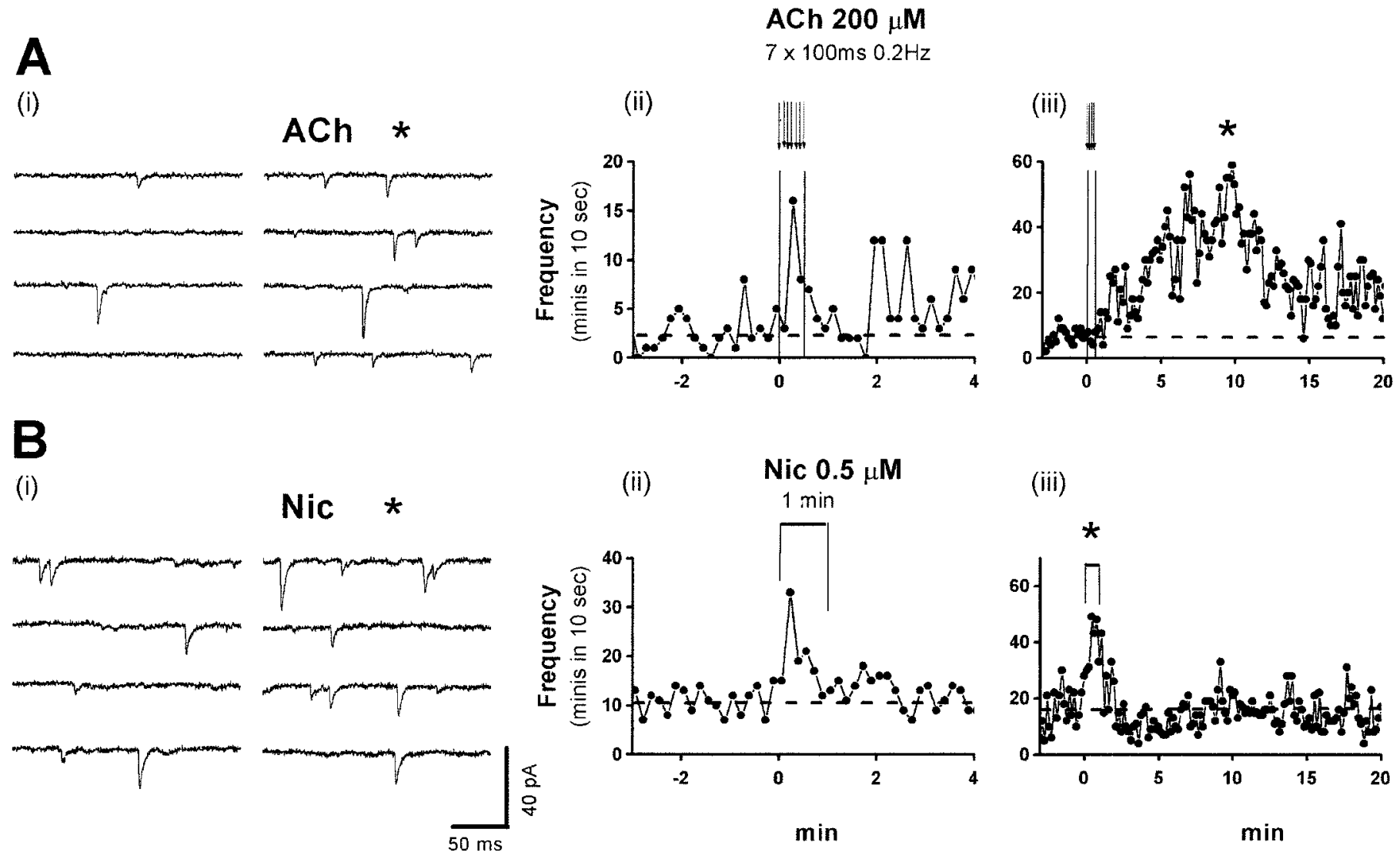

Figure 1. Facilitation of glutamatergic neurotransmission by synaptic-like pulses of acetylcholine and by "smokers" concentrations of nicotine. $A$, The electrophysiological traces in $A i$ depict glutamatergic minis recorded at an MHN-IPN synapse in culture, before and after the synapse had been challenged with seven $100 \mathrm{msec}$ pulses of $200 \mu \mathrm{M}$ ACh applied at $0.2 \mathrm{~Hz}$. The frequency plots in Aii and Aiii illustrate experiments performed at two distinct MHN-IPN synapses. They show the number of minis per $10 \mathrm{sec}$ intervals, before and after ACh application. Note the different time scales for the two plots. The recordings in $A i$ were taken from the experiment illustrated in Aiii. Asterisks indicate the time on the frequency plot at which the electrophysiological traces in $A i$ were sampled. Aii illustrates a synapse in which pulsatile ACh elicited immediate increase in the mini frequency, which was followed by a second episode of facilitation that lasted $\sim 10 \mathrm{~min}$. Aiii depicts a synapse in which facilitation was protracted and lasted up to 2 hr after ACh application. B, At two representative MHN-IPN synapses, application of a 1 min pulse of $0.5 \mu \mathrm{M}$ nicotine (Nic) enhanced the frequency of the minis. In Bii, the frequency peaked and then rapidly declined before the end of nicotine application, whereas in Biii, the frequency remained elevated during and up to $\sim 1 \mathrm{~min}$ after the end of the nicotine challenge. No protracted facilitation was seen with nicotine. The electrophysiological traces in $B i$ are taken from the experiment illustrated in Biii. Asterisks as in $A$.

The rise to peak facilitation and return to baseline mini frequency was relatively rapid with nicotine. Most nicotinefacilitated synapses reached peak frequency levels within 2 min of drug application (83\%) (Fig. 2Aii), and the average time-to-peak for nicotine-induced facilitation was $1.3 \pm 0.3 \mathrm{~min}$ (Fig. 2Bii). In contrast, only $50 \%$ of ACh-facilitated synapses reached a maximum increase in mini frequency within 2 min (Fig. 2 Aii), and the average time-to-peak facilitation was $7.0 \pm 2.7$ min (Fig. 2Bii), a value significantly larger than that found with nicotine.

Facilitation produced with ACh lasted much longer than facilitation induced by nicotine. With nicotine, the mini frequency remained elevated for $\sim 0.5-10 \mathrm{~min}$ after drug application. On average, the increase in mini frequency returned to baseline within $3.2 \pm 0.7 \mathrm{~min}$ of nicotine application (Fig. 2 Biii), and the duration of significant synaptic facilitation never exceeded $10 \mathrm{~min}$ (Fig. 2Aiii). In contrast, ACh-elicited facilitation lasted an average of $27.5 \pm 10.6 \mathrm{~min}$ (Fig. 2Biii). Transmission remained significantly elevated above control levels at $>40 \%$ of cholinoceptive synapses 10 min after ACh application. At $21 \%$ of cholinoceptive synapses, facilitation lasted for $>1.5-2 \mathrm{hr}$ (i.e., as long as we were able to reliably monitor activity) (Fig. 2Aiii,Ciii).

The facilitation elicited by ACh tended to be somewhat larger than that evoked by nicotine at most MHN-IPN connections. The average percentage change in frequency for ACh peaked at $557 \pm$ $159 \%$, whereas that for nicotine was $398 \pm 132 \%$ above the basal level of secretion (Fig. 2Biv). Although differences in the average facilitation elicited by $\mathrm{ACh}$ versus nicotine were not statistically significant, the cumulative frequency distributions are broad. Thus, $>70 \%$ of MHN-IPN synapses facilitated by nicotine were enhanced by less than twofold, whereas ACh elicited more than fivefold facilitation at the majority of synapses tested (Fig. 2Aiv).

Prolonged increases in mini frequency by $\mathrm{ACh}$ were also observed at synapses treated with the muscarinic antagonist atropine (Fig. 2Civ), consistent with a nicotinic receptor-mediated mechanism of sustained facilitation. Analysis of the subset of synapses that exhibited more rapid facilitation by $\mathrm{ACh}$ revealed an initial episode of facilitation, similar in magnitude and duration to that induced by nicotine, preceding the second phase of more sustained increased mini frequency (Fig. 2, compare $\mathrm{Ci}, \mathrm{Cii}$ ).

\section{Synaptic depression by muscarinic AChRs-mediated pathways}

At $21 \%$ of synapses assayed, ACh elicited a temporally distinct phase of synaptic depression during which a net decrease in mini 


\section{A \\ (i) Delay for onset of facilitation}
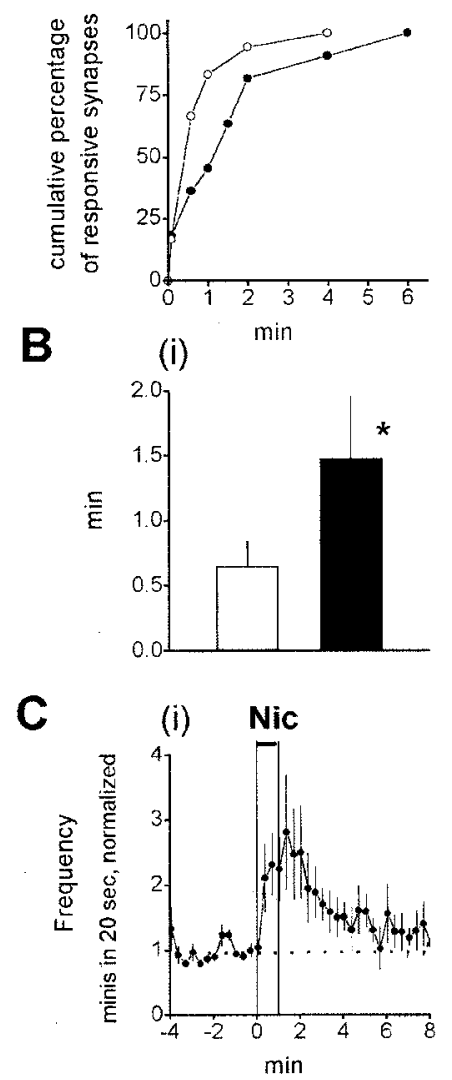

(ii) Time to max facilitation

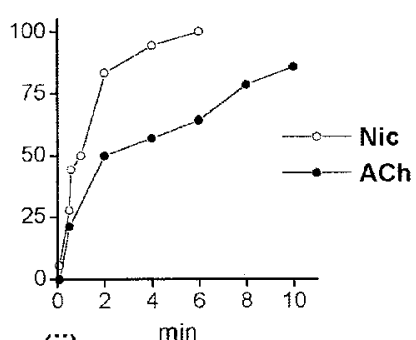

(ii)
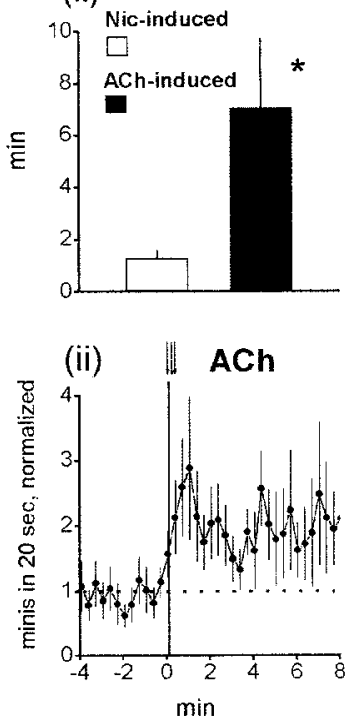

(iii) Duration of facilitation

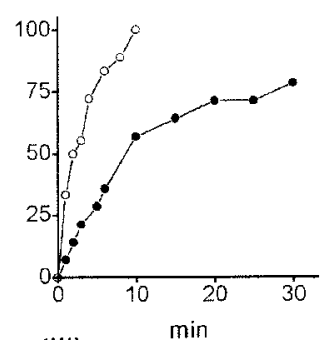

(iii)

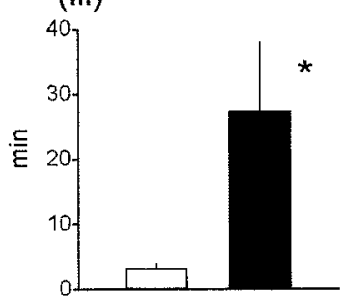

(iii) $₫ \mathrm{ACh}$

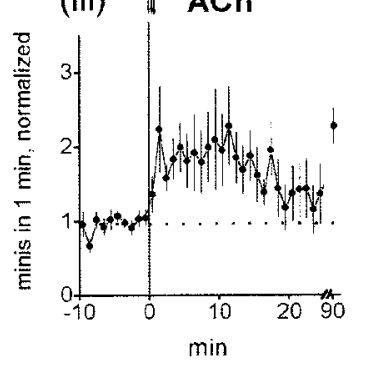

(iv) Max facilitation

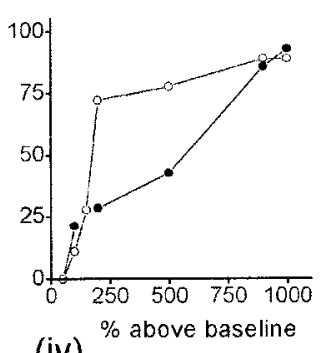

(iv)
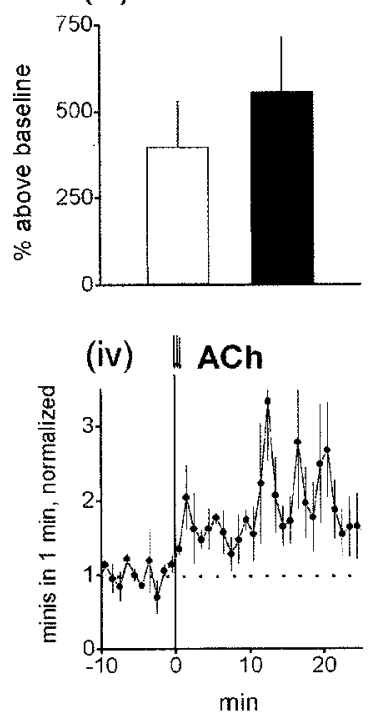

Atropine $0.5-1 \mu \mathrm{M}$

Figure 2. Quantitative analysis of the time course and magnitude of ACh- and nicotine-induced facilitation. $A$, The graphs depict cumulative histogram distributions of time course parameters and maximal value for ACh- and nicotine-induced facilitation. In $\mathrm{Ai}$, the delay for onset of facilitation is the time elapsed between the beginning of agonist application and the time when significant increase in mini frequency was observed. Each data point on the plot is the cumulative percentage of synapses for which the delay in minutes was shorter than or equal to a given value indicated on the abscissa. The other plots in Aii-Aiv were constructed in a similar manner. Right shifts of the curves indicate larger values of ACh-induced facilitation compared with nicotine-induced facilitation. These parameters were calculated at individual responsive synapses as described (see Materials and Methods). $B$, The column plots are average \pm SEM of the same parameters as those depicted in $A$. Asterisks indicate significant difference for nicotine versus ACh-induced facilitation. $C$, The graphs depict compound averages of normalized mini frequency measured at synapses that responded either to ACh or nicotine. For each synapse, the frequency was sampled as the number of minis occurring in $20 \mathrm{sec}$ bins in $\mathrm{Ci}$ and $\mathrm{Cii}$ and in $1 \mathrm{~min}$ bins in $\mathrm{Ciii}$ and $\mathrm{Civ}$. Before averaging, the frequency at individual synapses was normalized to the mean frequency observed before agonist application. Each data point depicts average \pm SEM normalized frequency. In $\mathrm{C} i$ is shown the average normalized frequency of 18 synapses (of a total of 44 synapses) that responded to 1 min of $0.5-1 \mu \mathrm{M}$ nicotine. In Cii is shown the average normalized frequency of eight synapses selected for their immediate response to seven $100 \mathrm{msec}$ pulses of $200 \mu \mathrm{M}$ $\mathrm{ACh}$ (delay for onset of facilitation $\leq 2 \mathrm{~min}$ ). In Ciii is shown average \pm SEM normalized frequency of all synapses (14 of a total of 24 synapses) that responded to ACh. The value at $90 \mathrm{~min}$ is the average of 3 of 14 responsive synapses that were still facilitated at this time. In $\mathrm{Civ}$ is depicted average \pm SEM normalized frequency of four synapses that responded to ACh (of a total of 8 synapses) in the presence of atropine $0.5-1 \mu \mathrm{M}$.

frequency was evident (Fig. 3). At this subset of synapses, mini frequency was decreased rapidly and repeatedly within seconds of ACh application. Recovery from inhibition was rapid, and activity returned to control within 10-20 sec after the end of ACh application (Fig. $3 A-C$ ). ACh-elicited inhibition of glutamate release was abolished by low concentrations of atropine $(500 \mathrm{~nm}$ to $1 \mu \mathrm{M}$ ) (Fig. $3 A, D$ ), and inhibition was never observed when nicotine was used as agonist. These data are consistent with inhibition of release by activation of presynaptic muscarinic receptors as reported previously (Marchi and Raiteri, 1989; Segal, 1989; Sheridan and Sutor, 1990; Vidal and Changeux, 1993; Barral et al., 1999; Rouse et al., 1999). At a subset of cholinoceptive synapses (13\%), ACh produced an initial decrease and then an increase in mini frequency (Fig. 3C). Concomitant inhibition and facilitation of synaptic transmission may arise from coordi- nate activation of presynaptic muscarinic and nicotinic AChRs on MHN inputs to IPN.

\section{Effects of prolonged exposure to nicotine}

The results presented above are consistent with the involvement of both nicotinic and muscarinic AChRs in the modulatory effects of ACh at MHN-IPN synapses. Hence, the net effect of ACh will depend (at least in part) on the relative contribution of these two general classes of ACh receptors at individual synapses. Continuous exposure to low-level nicotine can cause reversible desensitization, permanent inactivation, and increase in the number of surface nAChRs. The extent of these effects depends on the receptor subtypes and the cell type in which the receptors are expressed (Wonnacott, 1990; Peng et al., 1994; Dani and Heinemann, 1996; Olale et al., 1997; Peng et al., 1997; Wang et al., 1998; 
Figure 3. Inhibition of glutamatergic neurotransmission by synaptic-like pulses of acetylcholine. $A$, The frequency plot depicts an experiment in which seven 100 msec pulses of ACh $200 \mu \mathrm{M}$ given at $0.2 \mathrm{~Hz}$ produced transient inhibition of synaptic transmission at an MHN-IPN synapse. A second round of ACh stimulation applied in the presence of $1 \mu \mathrm{M}$ atropine failed to inhibit transmission. $B$, The electrophysiological traces were sampled from the experiment shown in $A$. They illustrate minis recorded before (top 2 traces), during (middle 2 traces), and after (bottom 2 traces) application of ACh in the absence of atropine. $C$, Average normalized mini frequency of four synapses (of a total of 24 synapses examined) that were inhibited by the pulses of ACh. Note that, at the end of the episode of inhibition, facilitation became apparent, indicating the contribution of both nicotinic and muscarinic receptors. Frequency bin, $10 \mathrm{sec}$. $D$, Average normalized mini frequency of eight synapses that were recorded in the presence of 0.5-1 $\mu \mathrm{M}$ atropine. None of these synapses exhibited detectable synaptic inhibition and were thus averaged for the plot. Some nicotinic synaptic facilitation was apparent during the pulses of ACh.
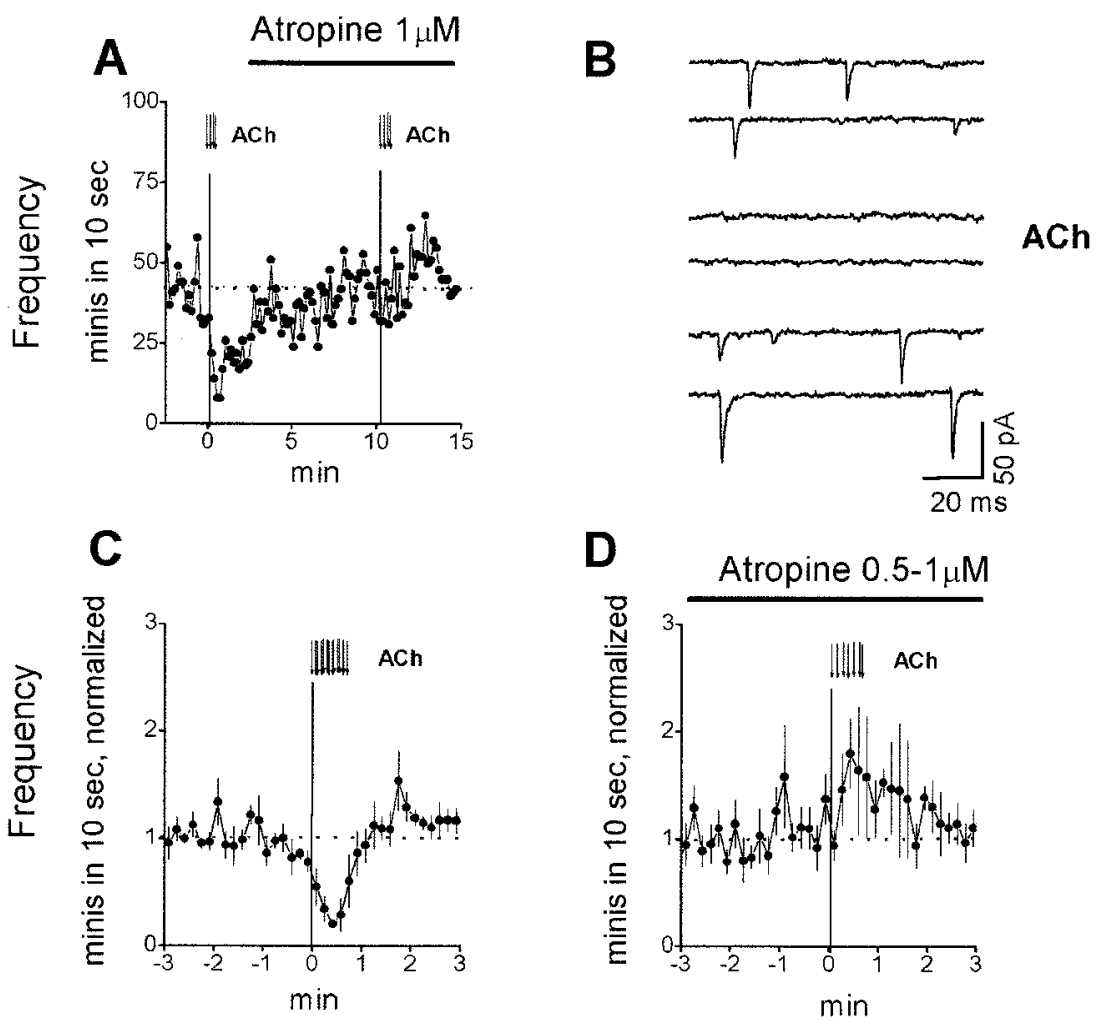

Kuryatov et al., 2000). To examine the net effects of continuous nicotine on responses to subsequent application of nicotine and ACh, we exposed MHN-IPN synapses to nicotine for sustained periods of time as described in Materials and Methods. Synaptic transmission in response to subsequent challenges with either acute nicotine or ACh was assayed within the first $3 \mathrm{hr}$ after removal of the drug and replacement in nicotine-free recording media. In this set of experiments, we calculated the average rate of responsiveness to nicotinic agonists within platings as described in Figure 4. In a subset of control cultures, an average of $51 \pm 6 \%$ synapses was thus found to respond to acute nicotine (Fig. $4 A, G$; an evaluation of between-sample vs within-sample variance performed on the entire pool of synaptic connections compared with that of this subset of connections revealed no significant difference). After prolonged exposure to nicotine, the average rate of responsiveness was significantly reduced (19 \pm $8 \%$ ) (Fig. 4B, $G$ ).

Because recovery of presynaptic facilitation did not occur within the first $3 \mathrm{hr}$ after nicotine treatment, we tested whether longer recovery times in nicotine-free medium would return responsiveness to pretreatment levels. Synaptic cocultures, previously exposed to nicotine for $48 \mathrm{hr}$, were allowed to recover in control medium for $>3-24 \mathrm{hr}$ before assay (Fig. 4G). After $\sim 5 \mathrm{hr}$ in a nicotine-free environment, there was little recovery of nicotine-elicited synaptic facilitation or of the percentage of nicotine-responsive synapses. Full recovery to "pretreatment" control levels of nicotine-induced synaptic facilitation required 24-27 hr of "drug-free" conditions (percentage of nicotineresponsive synapses, $48 \pm 8$ vs $51 \pm 6 \%$ in control).

Facilitatory responses to ACh (of both short and long duration) were similarly susceptible to the apparent inactivation of nicotinic AChRs by prolonged exposure to nicotine. ACh-elicited increases in mini frequency were detected at only $19 \pm 12 \%$ of MHN-IPN synapses subjected to prolonged nicotine treatment (compared with $60 \pm 14 \%$ of sibling control synapses) (Fig. $4 C, D, H$ ). In contrast, muscarinic AChR-mediated effects were unaltered by nicotine exposure. Thus, the temporal profile and extent of synaptic inhibition elicited by $\mathrm{ACh}$ was equivalent in control and nicotine-treated conditions (Fig. $4 E, F, H$ ). The net effect of prolonged nicotine exposure, therefore, is a significant decrease in the proportion of synapses that are facilitated versus those that are depressed by subsequent challenges with ACh (Fig. 4H).

Sustained nicotine was without significant effect on other parameters of glutamatergic synaptic transmission at MHN-IPN synapses. Neither the basal frequency, average amplitude, amplitude distribution, nor rise or decay time of glutamatergic minis were altered by prolonged exposure to nicotine (Table 1). Likewise, nicotine did not significantly affect either the amplitude or kinetics of currents recorded in IPN neurons in response to direct application of glutamate $(300 \mu \mathrm{M})$ (Table 1$)$.

\section{DISCUSSION}

$\mathrm{ACh}$, delivered in repetitive high-concentration pulses, can elicit both facilitatory and inhibitory neuromodulation of glutamatergic synapses between MHN and IPN neurons. Initially, ACh-elicited facilitation matches the typically monophasic and short-lived effects of nicotine delivered as when inhaled through smoking. Second, and perhaps most importantly, ACh can elicit sustained enhancement of glutamate transmission, an effect that also appears to be initiated by interaction with nicotinic AChRs. At most cholinoceptive synapses ACh elicited facilitation that lasted for $\sim 30 \mathrm{~min}$. At $21 \%$ of responsive synapses facilitation was persistent, lasting for $>1 \mathrm{hr}$ after application of a single set of ACh pulses. Finally, phasic application of $\mathrm{ACh}$, but not nicotine, also elicits significant inhibition of synaptic transmission at a subset of MHN-IPN synapses. Synaptic inhibition appears to involve the activation of muscarinic AChRs and was relatively transient. 


\section{Control}

A
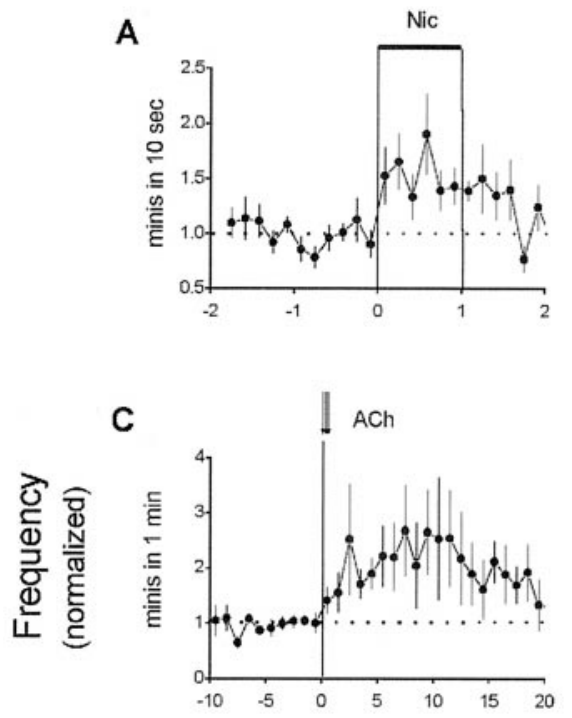

E

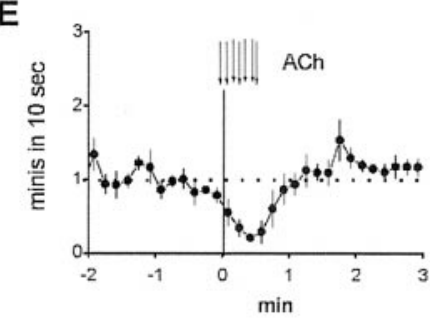

G

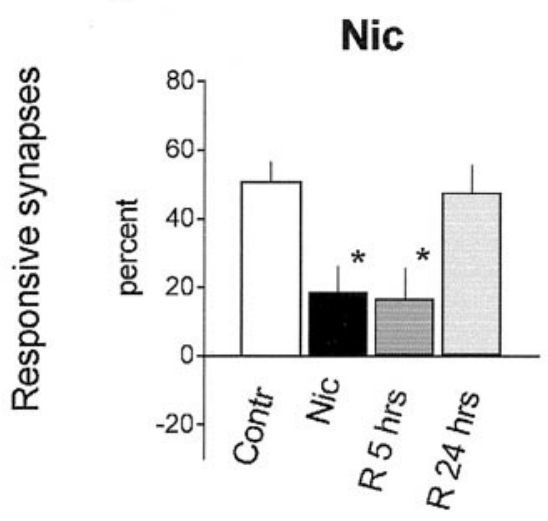

B

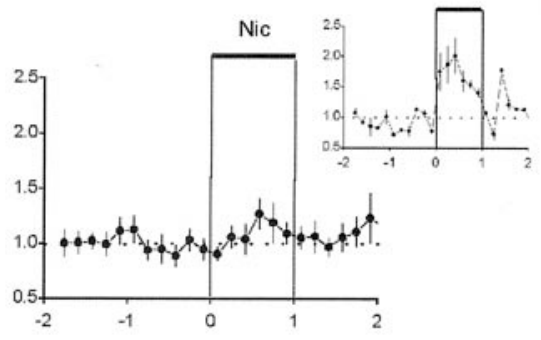

D

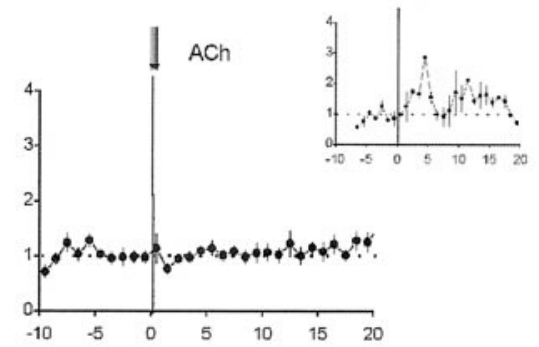

$\mathbf{F}$

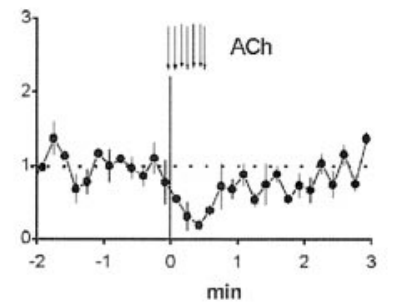

$\mathrm{H}$

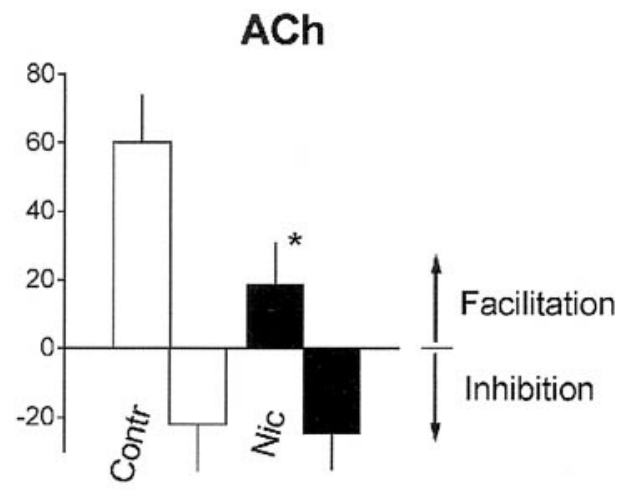

Figure 4. Prolonged exposure to nicotine inactivates presynaptic nicotinic AChRs but does not affect muscarinic receptors. The frequency plots illustrate recordings performed in control conditions and after prolonged exposure to nicotine. Note the different time scales in these graphs. They depict average normalized frequency: $A$, 12 control synapses that responded to acute nicotine; $B, 19$ synapses in which acute nicotine failed to elicit synaptic modulation after prolonged treatment with nicotine (in the inset are depicted 5 treated synapses that responded to acute nicotine); $C, 10$ control synapses that responded to pulsatile application of $\mathrm{ACh}$; $D, 12$ treated synapses that were unresponsive to $\mathrm{ACh}$ (the inset illustrates 3 treated synapses that exhibited responsiveness to Ach); $E$, four control synapses that were inhibited by $\mathrm{ACh}$; and $F$, three synapses treated with prolonged nicotine that were inhibited by ACh. Note that, in contrast to control synapses $(E)$, some level of facilitation did not follow inhibition after prolonged exposure to nicotine. In $G$ and $H$, the data are shown as percentage of synapses that were modulated by nicotine or ACh, respectively. Columns depict the average \pm SEM percentage of synapses facilitated by nicotine and either facilitated or inhibited by ACh under the following conditions: in control coculture (Contr); after $24-72 \mathrm{hr}$ treatment with 1 $\mu \mathrm{M}$ nicotine (Nic); after $48 \mathrm{hr}$ treatment with the drug followed by $5 \mathrm{hr}$ recovery in nicotine-free media $(R 5 \mathrm{hr})$; and after 48 hr treatment with the drug, followed by 24 hr recovery $(R 24 h r)$. To calculate the average percentage of responsive synapses, the number of synapses that responded to nicotine or ACh was divided by the total number of recorded synapses in each culture plating. Then, the ratios in three to six different culture platings for each condition were averaged. For convenience, upward columns illustrate the percentage of synapses that were facilitated in each condition, whereas downward columns illustrate the percentage of synapses that were inhibited.

\section{Nicotinic ACh receptor-mediated facilitation}

Our protocol for ACh delivery was intended to mimic secretion of ACh from active cholinergic nerve endings. Obviously, the exact profile of agonist exposure experienced by cholinergic receptive sites in the IPN is unknown and our protocol reflects a "bestguess" based on limited in vivo data on central cholinergic activity. Phasic, rather than continuous, application of ACh is likely to better approximate $\mathrm{ACh}$ release during activation of cholinergic neurons. Different subsets of cholinergic neurons have been found to discharge intermittent bursts of action potentials (Khateb et al., 1992; Alonso et al., 1996; Manns et al., 2000), and ACh released from cholinergic nerve endings is rapidly degraded by acetylcholinesterase. Morphological evidence consistent with a presynaptic or perisynaptic release of $\mathrm{ACh}$ has been provided in cortical and limbic areas of the brain (Woolf, 1991; Lubin et al., 1999). Pulsatile delivery of ACh producing persistent facilitation of glutamatergic transmission likely occurs at sites of MHN-IPN contact in vivo, because the IPN receives the most abundant cholinergic innervation in the brain, arising from cholinergic nuclei located within the basal forebrain, the brainstem, and from cholinergic neurons within the MHN per se (Woolf, 1991).

This study also attempted to model the effects of nicotine because it may be delivered with both "acute" (i.e., when smoking a single cigarette) and prolonged (i.e., in habitual smokers) ad- 
Table 1. Prolonged nicotine did not alter basal spontaneous glutamate secretion

\begin{tabular}{|c|c|c|c|c|c|}
\hline & \multicolumn{4}{|c|}{ Basal secretion of glutamatergic minis } & \multirow{2}{*}{$\begin{array}{l}\begin{array}{l}\text { Response to } 300 \mu \mathrm{M} \\
\text { glutamate }\end{array} \\
\begin{array}{l}\text { Average peak current } \\
\text { (pA) }\end{array}\end{array}$} \\
\hline & $\begin{array}{l}\text { Average frequency } \\
(\text { minis/5 sec) }\end{array}$ & $\begin{array}{l}\text { Average amplitude } \\
\text { (pA) }\end{array}$ & $\begin{array}{l}\text { Average rise time } \\
(\mathrm{msec})\end{array}$ & $\begin{array}{l}\text { Average decay time } \\
\text { (msec) }\end{array}$ & \\
\hline Control & $10.1 \pm 2.1$ & $13.0 \pm 0.8$ & $1.2 \pm 0.1$ & $3.6 \pm 0.7$ & $319 \pm 43$ \\
\hline Prolonged nicotine & $6.6 \pm 1.4$ & $13.2 \pm 0.9$ & $1.3 \pm 0.1$ & $4.2 \pm 0.6$ & $272 \pm 44$ \\
\hline Recovery $5 \mathrm{hr}$ & $6.3 \pm 2.3$ & $12.3 \pm 1.7$ & $1.1 \pm 0.1$ & $3.1 \pm 0.6$ & ND \\
\hline Recovery $24 \mathrm{hr}$ & $6.8 \pm 1.9$ & $13.8 \pm 1.6$ & $1.2 \pm 0.2$ & $2.7 \pm 0.3$ & ND \\
\hline
\end{tabular}

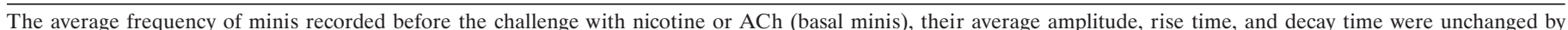

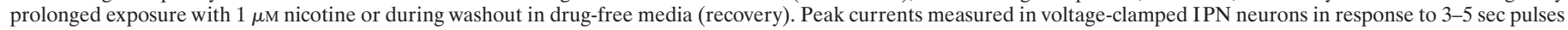
of $300 \mu \mathrm{M}$ glutamate were not affected by prolonged nicotine exposure. ND, Not determined.

ministration by cigarette smoking. Smoking results in gradual increases and decreases of arterial nicotine concentration, which are likely to sum with repeated administrations (i.e., with each "puff" and with multiple cigarettes) because the turnover of nicotine is relatively slow. The maximal drug concentrations achieved are thought to be in the range of 0.5-1 $\mu \mathrm{M}$ (Benowitz et al., 1989; Russell, 1989; Henningfield et al., 1993). Acute smoking was modeled by applying $0.5-1 \mu \mathrm{M}$ nicotine to MHN-IPN synapses continuously for $1 \mathrm{~min}$. The drug elicited synaptic facilitation that was relatively short lived and that subsided within minutes of drug removal (McGehee et al., 1995; Alkondon et al., 1996; Gray et al., 1996; Guo et al., 1998; present study).

The most parsimonious interpretation of our results is that acute nicotine and the pulses of ACh initiate facilitation by similar cellular mechanisms, that is, by activating presynaptic nAChRs expressed at MHN nerve terminals (McGehee et al., 1995). The following observations support this view: (1) ACh enhanced the frequency of minis without changing their amplitude; (2) sustained ACh-induced facilitation persisted in the presence of the muscarinic antagonist atropine; (3) both AChand nicotine-induced facilitation were depressed to a similar extent when $\mathrm{nAChRs}$ were deactivated by preexposure with nicotine; and (4) ACh can elicit an initial episode of facilitation with time course and magnitude that closely resemble those of the short burst of facilitation induced by nicotine.

Based on these findings and those of numerous other studies, activation of presynaptic nAChRs, by either ACh or nicotine, appears to promote calcium influx, indirectly through voltage gated calcium channels and/or directly through the nicotinic receptors (Mulle et al., 1992; McGehee et al., 1995; Gray et al., 1996; Lena and Changeux, 1997; Girod et al., 2000). The nAChRelicited increase in intraterminal calcium thereby initiates presynaptic facilitation, whereas the more prolonged facilitation observed with "synaptic-like" application of ACh might arise from downstream effects, such as calcium-induced calcium release and subsequent activation of second-messenger cascade(s). In addition, other mechanisms involved in long-term potentiation of glutamatergic synapses (Mansvelder and McGehee, 2000) may account for the prolonged changes in synaptic transmission reported here. If the effects of $\mathrm{ACh}$ involve the activation of NMDA receptors, then inhibition of these receptors as a result of nAChR activation (Fisher and Dani, 2000) may work as a negative feedback mechanism to limit synaptic responses to ACh.

Pulsatile application of high concentrations of nicotine recapitulates some of the more long-lasting effects of ACh, inducing facilitation for 5-10 min in hippocampal cultures (Radcliffe and Dani, 1998). Persistent enhancement of glutamate release lasting
$1 \mathrm{hr}$ or more, initiated by ACh and mediated via nicotinic receptor activation, has not, to our knowledge, been reported previously. Although our results are consistent with ACh causing prolonged enhancement of evoked secretion of glutamate, such long-term effects could not be investigated in the present system because stable recordings of evoked transmission (i.e., $>10 \mathrm{~min}$ ) are rare.

Recent reports have suggested that nicotine can facilitate the induction of long-term potentiation of glutamatergic transmission in the hippocampus (Fujii et al., 1999) and in the midbrain dopaminergic reward pathways (Mansvelder and McGehee, 2000). The present results show that the endogenous neurotransmitter ACh may use similar cellular mechanisms to promote sustained facilitation of glutamate secretion via activation of presynaptic nAChRs.

\section{Muscarinic ACh receptor-mediated inhibition}

Our observation that synaptic-like pulses of ACh elicited muscarinic receptor-mediated inhibition of glutamate release at $\mathrm{MHN}-$ IPN synapses is consistent with data obtained in other brain regions, including the hippocampus (Marchi and Raiteri, 1989; Segal, 1989; Sheridan and Sutor, 1990; Rouse et al., 1999), striatum (Barral et al., 1999), and cortex (Vidal and Changeux, 1993). Results obtained in the hippocampus (Egorov et al., 1996; Qian and Saggau, 1997) and in the striatum (Barral et al., 1999) indicate that synaptic depression results from the inhibition of voltage-gated calcium channels by presynaptic mAChRs. A direct interaction of mAChRs with the secretory machinery has also been proposed (Scanziani et al., 1995; Linial et al., 1997).

\section{Prolonged exposure with nicotine may alter the balance between the facilitatory and inhibitory effects of $\mathrm{ACh}$}

ACh may facilitate or inhibit transmission at MHN-IPN synapses, depending on whether the ligand interacts with presynaptic nicotinic and/or muscarinic AChRs. Opposing effects of ACh by activation of $\mathrm{nAChRs}$ versus $\mathrm{mAChRs}$ have also been reported in studies of cortical synapses (Vidal and Changeux, 1993). At such sites, the balance of synaptic facilitation versus depression, and hence, the net effect of ACh, will be determined by the relative contribution of functional nicotinic versus muscarinic AChRs. Shifts of this balance may occur in habitual smokers in which receptive sites are exposed to sustained, low levels of nicotine that are expected to selectively desensitize or inactivate certain subtypes of nAChRs (Wonnacott, 1990; Peng et al., 1994; Dani and Heinemann, 1996; Olale et al., 1997; Peng et al., 1997; Wang et al., 1998; Kuryatov et al., 2000). Habitual smokers seem to adjust 
their cigarette consumption so as to achieve stable plasma concentration of nicotine throughout the day (Benowitz et al., 1989; Russell, 1989; Henningfield et al., 1993). We modeled habitual smoking by exposing MHN-IPN cultures to nicotine for extended periods of time (24-72 hr). Prolonged exposure to nicotine depressed both nicotine- and ACh-mediated presynaptic facilitation, without altering muscarinic receptor-mediated synaptic depression, consistent with a functional inactivation of presynaptic nAChRs. Full recovery of nicotinic receptormediated synaptic facilitation required $\sim 24 \mathrm{hr}$, suggesting an approximate half-time for recovery of $\sim 10-12 \mathrm{hr}$. Such slow kinetics are consistent with proposed models of sustained deactivation of nAChRs, requiring new receptor synthesis and insertion for full recovery of function. Similar effects of prolonged exposure to nicotine were found for $\mathrm{nAChRs}$ expressed in reconstituted systems (Whiting et al., 1991; Peng et al., 1994, 1997). Because muscarinic receptor activation and ACh-elicited presynaptic inhibition are unaltered by prolonged exposure to nicotine, the treatment shifts the balance toward muscarinic AChRmediated inhibition and decreases the contribution of nicotinic AChR-mediated presynaptic facilitation.

Our protocol for prolonged exposure to nicotine only partially recapitulates habitual smoking. In contrast to continuous treatment with the drug, a typical day of smoking is interrupted by several hours of smoke-free sleep. Nevertheless, indirect evidence suggests that, despite nocturnal interruption of nicotine selfadministration, smoking induces significant inactivation of brain nicotinic receptor. Indeed, the number of nicotinic receptor binding sites in postmortem brains increases in habitual smokers (Breese et al., 1997; Perry et al., 1999), and surface receptor upregulation has been proposed to be initiated by inactivation of receptors by chronic nicotine (Marks et al., 1983; Schwartz and Kellar, 1985; Fenster et al., 1999). Our results suggest that inactivation of certain subtypes of nicotinic receptor by nicotine inhaled through smoking may cause a switch between presynaptic facilitatory and inhibitory influences of central cholinergic projections.

Overall, these results suggest that activation of central cholinergic pathways may regulate neuronal networks by producing a combination of long-lasting presynaptic nicotinic AChRmediated facilitation and shorter-term, presynaptic muscarinic AChR-mediated inhibition of glutamatergic neurotransmission. Furthermore, aspects of nicotine dependence may involve selective disruption of the nicotinic component of cholinergic signaling by prolonged exposure to nicotine.

\section{REFERENCES}

Alkondon M, Rocha ES, Maelicke A, Albuquerque EX (1996) Diversity of nicotinic acetylcholine receptors in rat brain. V. alpha-Bungarotoxinsensitive nicotinic receptors in olfactory bulb neurons and presynaptic modulation of glutamate release. J Pharmacol Exp Ther 278:1460-1471.

Alonso A, Khateb A, Fort P, Jones BE, Muhlethaler M (1996) Differential oscillatory properties of cholinergic and noncholinergic nucleus basalis neurons in guinea pig brain slice. Eur J Neurosci 8:169-182.

Barral J, Galarraga E, Bargas J (1999) Muscarinic presynaptic inhibition of neostriatal glutamatergic afferents is mediated by Q-type $\mathrm{Ca}^{2+}$ channels. Brain Res Bull 49:285-289.

Benowitz NL, Porchet H, Jacob III P (1989) Nicotine dependence and tolerance in man: pharmacokinetic and pharmacodynamic investigations. Prog Brain Res 79:279-287.

Breese CR, Marks MJ, Logel J, Adams CE, Sullivan B, Collins AC, Leonard S (1997) Effect of smoking history on $\left[{ }^{3} \mathrm{H}\right]$ nicotine binding in human postmortem brain. J Pharmacol Exp Ther 282:7-13.

Brown DA, Docherty RJ, Halliwell JV (1983) Chemical transmission in the rat interpeduncular nucleus in vitro. J Physiol (Lond) 341:655-670.

Brussaard AB, Yang X, Doyle JP, Huck S, Role LW (1994) Develop- mental regulation of multiple nicotinic $\mathrm{nAChR}$ channel subtypes in embryonic chick habenula neurons: contributions of both the $\alpha 2$ and $\alpha 4$ subunit genes. Pflügers Arch 429:27-43.

Dani JA, Heinemann S (1996) Molecular and cellular aspects of nicotine abuse. Neuron 16:905-908.

Egorov AV, Heinemann U, Muller W (1996) Muscarinic activation reduces changes in $\left[\mathrm{Ca}^{2+}\right]$ o evoked by stimulation of Schaffer collaterals during blocked synaptic transmission in rat hippocampal slices. Neurosci Lett 214:187-190.

Fenster CP, Whitworth TL, Sheffield EB, Quick MW, Lester RA (1999) Upregulation of surface $\alpha 4 \beta 2$ nicotinic receptors is initiated by receptor desensitization after chronic exposure to nicotine. J Neurosci 19:4804-4814.

Fisher JL, Dani JA (2000) Nicotinic receptors on hippocampal cultures can increase synaptic glutamate currents while decreasing the NMDAreceptor component. Neuropharmacology 39:2756-2769.

Fujii S, Ji Z, Morita N, Sumikawa K (1999) Acute and chronic nicotine exposure differentially facilitate the induction of LTP. Brain Res 846:137-143.

Gardette R, Listerud MD, Brussaard AB, Role LW (1991) Developmental changes in transmitter sensitivity and synaptic transmission in embryonic chicken sympathetic neurons innervated in vitro. Dev Biol 147:83-95.

Girod R, Barazangi N, McGehee D, Role LW (2000) Facilitation of glutamatergic neurotransmission by presynaptic nicotinic acetylcholine receptors. Neuropharmacology 39:2715-2725.

Grady S, Marks MJ, Wonnacott S, Collins AC (1992) Characterization of nicotinic receptor-mediated $\left[{ }^{3} \mathrm{H}\right]$ dopamine release from synaptosomes prepared from mouse striatum. J Neurochem 59:848-856.

Gray R, Rajan AS, Radcliffe KA, Yakehiro M, Dani JA (1996) Hippocampal synaptic transmission enhanced by low concentrations of nicotine. Nature 383:713-716.

Guo JZ, Tredway TL, Chiappinelli VA (1998) Glutamate and GABA release are enhanced by different subtypes of presynaptic nicotinic receptors in the lateral geniculate nucleus. J Neurosci 18:1963-1969.

Hamill OP, Marty A, Neher E, Sakmann B, Sigworth FJ (1981) Improved patch-clamp techniques for high-resolution current recording from cells and cell-free membrane patches. Pflügers Arch 391:85-100.

Henningfield JE, Stapleton JM, Benowitz NL, Grayson RF, London ED (1993) Higher levels of nicotine in arterial than in venous blood after cigarette smoking. Drug Alcohol Depend 33:23-29.

Horn R, Marty A (1988) Muscarinic activation of ionic currents measured by a new whole-cell recording method. J Gen Physiol 92:145-159.

Jones S, Sudweeks S, Yakel JL (1999) Nicotinic receptors in the brain: correlating physiology with function. Trends Neurosci 22:555-561.

Khateb A, Muhlethaler M, Alonso A, Serafin M, Mainville L, Jones BE (1992) Cholinergic nucleus basalis neurons display the capacity for rhythmic bursting activity mediated by low-threshold calcium spikes. Neuroscience 51:489-494.

Kuryatov A, Olale FA, Choi C, Lindstrom J (2000) Acetylcholine receptor extracellular domain determines sensitivity to nicotine-induced inactivation. Eur J Pharmacol 393:11-21.

Lena C, Changeux JP (1997) Role of $\mathrm{Ca}^{2+}$ ions in nicotinic facilitation of GABA release in mouse thalamus. J Neurosci 17:576-585.

Levin ED (1992) Nicotinic systems and cognitive function. Psychopharmacology 108:417-431.

Linial M, Ilouz N, Parnas H (1997) Voltage-dependent interaction between the muscarinic ACh receptor and proteins of the exocytic machinery. J Physiol (Lond) 504:251-258.

Lubin M, Erisir A, Aoki C (1999) Ultrastructural immunolocalization of the alpha $7 \mathrm{nAChR}$ subunit in guinea pig medial prefrontal cortex. Ann NY Acad Sci 868:628-632.

MacDermott AB, Role LW, Siegelbaum SA (1999) Presynaptic ionotropic receptors and the control of transmitter release. Annu Rev Neurosci 22:443-485.

Manns ID, Alonso A, Jones BE (2000) Discharge properties of juxtacellularly labeled and immunohistochemically identified cholinergic basal forebrain neurons recorded in association with the electroencephalogram in anesthetized rats. J Neurosci 20:1505-1518.

Mansvelder HD, McGehee DS (2000) Long-term potentiation of excitatory inputs to brain reward areas by nicotine. Neuron 27:349-357.

Marchi M, Raiteri M (1989) Interaction acetylcholine-glutamate in rat hippocampus: involvement of two subtypes of M-2 muscarinic receptors. J Pharmacol Exp Ther 248:1255-1260.

Marks MJ, Burch JB, Collins AC (1983) Effects of chronic nicotine infusion on tolerance development and nicotinic receptors. J Pharmacol Exp Ther 226:817-825.

McGehee DS, Role LW (1996) Presynaptic ionotropic receptors. Curr Opin Neurobiol 6:342-349.

McGehee D, Heath M, Gelber S, Devay P, Role LW (1995) Nicotine enhancement of fast excitatory synaptic transmission in CNS by presynaptic receptors. Science 269:1692-1697.

Mulle C, Choquet D, Korn H, Changeux J-P (1992) Calcium influx through nicotinic receptor in rat central neurons: its relevance to cellular regulation. Neuron 8:135-143. 
Olale F, Gerzanich V, Kuryatov A, Wang F, Lindstrom J (1997) Chronic nicotine exposure differentially affects the function of human alpha3, alpha4, and alpha7 neuronal nicotinic receptor subtypes. J Pharmacol Exp Ther 283:675-683.

Peng X, Gerzanich V, Anand R, Whiting PJ, Lindstrom J (1994) Nicotine-induced increase in neuronal nicotinic receptors results from a decrease in the rate of receptor turnover. Mol Pharmacol 46:523-530.

Peng X, Gerzanich V, Anand R, Wang F, Lindstrom J (1997) Chronic nicotine treatment up-regulates alpha3 and alpha7 acetylcholine receptor subtypes expressed by the human neuroblastoma cell line $\mathrm{SH}$ SY5Y. Mol Pharmacol 51:776-784.

Perry DC, Davila-Garcia MI, Stockmeier CA, Kellar KJ (1999) Increased nicotinic receptors in brains from smokers: membrane binding and autoradiography studies. J Pharmacol Exp Ther 289:1545-1552.

Qian J, Saggau P (1997) Presynaptic inhibition of synaptic transmission in the rat hippocampus by activation of muscarinic receptors: involvement of presynaptic calcium influx. Br J Pharmacol 122:511-519.

Radcliffe KA, Dani JA (1998) Nicotinic stimulation produces multiple forms of increased glutamatergic synaptic transmission. J Neurosci 18:7075-7083.

Rae J, Cooper K, Gates P, Watsky M (1991) Low access resistance perforated patch recordings using amphotericin B. J Neurosci Methods 37:15-26.

Rapier C, Lunt GG, Wonnacott S (1990) Nicotine modulation of $\left[{ }^{3} \mathrm{H}\right]$ dopamine release from striatal synaptosomes: pharmacological characterization. J Neurochem 54:937-945.

Role L, Berg DK (1996) Nicotinic receptors in the development and modulation of CNS synapses. Neuron 16:1077-1085.

Role LW (1988) Neural regulation of acetylcholine sensitivity in embryonic sympathetic neurons. Proc Natl Acad Sci USA 85:2825-2829.

Rose JE, Corrigall WA (1997) Nicotine self-administration in animals and humans: similarities and differences. Psychopharmacology (Berl) 130:28-40.

Rouse ST, Marino MJ, Potter LT, Conn PJ, Levey AI (1999) Muscarinic receptor subtypes involved in hippocampal circuits. Life Sci 64:501-509.

Russell MA (1989) Subjective and behavioural effects of nicotine in humans: some sources of individual variation. Prog Brain Res 79:289-302.

Sarter M, Bruno JP (1997) Cognitive functions of cortical acetylcholine: toward a unifying hypothesis. Brain Res Rev 23:28-46.

Scanziani M, Gahwiler BH, Thompson SM (1995) Presynaptic inhibition of excitatory synaptic transmission by muscarinic and metabotropic glutamate receptor activation in the hippocampus: are $\mathrm{Ca}^{2+}$ channels involved? Neuropharmacology 34:1549-1557.

Schelling TC (1992) Addictive drugs: the cigarette experience. Science 255:430-433.

Schwartz RD, Kellar KJ (1985) In vivo regulation of $\left[{ }^{3} \mathrm{H}\right]$ acetylcholine recognition sites in brain by nicotinic cholinergic drugs. J Neurochem 45:427-433.

Segal M (1989) Presynaptic cholinergic inhibition in hippocampal cultures. Synapse 4:305-312.

Sheridan RD, Sutor B (1990) Presynaptic M1 muscarinic cholinoceptors mediate inhibition of excitatory synaptic transmission in the hippocampus in vitro. Neurosci Lett 108:273-278.

Stolerman IP, Jarvis MJ (1995) The scientific case that nicotine is addictive. Psychopharmacology (Berl) 117:2-10; discussion 14-20.

Stolerman IP, Shoaib M (1991) The neurobiology of tobacco addiction. Trends Pharmacol Sci 12:467-473.

Vidal C, Changeux JP (1993) Nicotinic and muscarinic modulations of excitatory synaptic transmission in the rat prefrontal cortex in vitro. Neuroscience 56:23-32.

Wainer BH, Steininger TL, Roback JD, Burke-Watson MA, Mufson EJ, Kordower J (1993) Ascending cholinergic pathways: functional organization and implications for disease models. Prog Brain Res 98:9-30.

Wang F, Nelson ME, Kuryatov A, Olale F, Cooper J, Keyser K, Lindstrom J (1998) Chronic nicotine treatment up-regulates human alpha3 beta 2 but not alpha3 beta4 acetylcholine receptors stably transfected in human embryonic kidney cells. J Biol Chem 273:28721-28732.

Whiting P, Schoepfer R, Lindstrom J, Priestley T (1991) Structural and pharmacological characterization of the major brain nicotinic acetylcholine receptor subtype stably expressed in mouse fibroblasts. Mol Pharmacol 40:463-472.

Wilson JR, Mitchell JC, Van Hoesen GW (1972) Epithalamic and ventral tegmental contributions to avoidance behavior in rats. J Comp Physiol Psychol 78:442-449.

Wirtshafter D (1981) The role of interpeduncular connections with the tegmentum in avoidance learning. Physiol Behav 26:985-989.

Wonnacott S (1990) The paradox of nicotinic acetylcholine receptor upregulation by nicotine. Trends Pharmacol Sci 11:216-219.

Wonnacott S (1997) Presynaptic nicotinic ACh receptors. Trends Neurosci 20:92-98.

Woolf NJ (1991) Cholinergic systems in mammalian brain and spinal cord. Prog Neurobiol 37:475-524. 